\title{
Migración y renovación generacional en la agricultura familiar indígena: estudio de caso Otavalo-Ecuador
}

\section{Migration and generational renewal in the indigenous family farming: case study Otavalo-Ecuador}

\author{
David Eche ${ }^{1}$ \\ 凶:mdeche@uce.edu.ec. \\ 1 Carrera Ingeniería Agronómica, Facultad de Ciencias Agrícolas, Universidad Central del Ecuador,
}

\begin{abstract}
Resumen
El presente proyecto analiza la migración y la renovación generacional en la agricultura familiar indígena como parte del proceso de la desagrarización, desde los enfoques de la agricultura familiar indígena y la juventud rural en el cantón indígena kichwa de Otavalo. Mediante análisis cuantitativos y cualitativos de los datos obtenidos con encuestas estructuradas, se determinó que este tipo de agricultura adolece de severos problemas de productividad que afectan negativamente sobre la generación de ingresos económicos, los cuales fomentan la emigración rural. Particularmente, la disminución de la productividad de la agricultura familiar se debe a factores ambientales como la escasez de agua y la degradación de suelos. El abandono de la actividad agrícola esta principalmente relacionada con la falta de apoyos económicos y técnicos gubernamentales a la agricultura familiar, forzando a los agricultores a la búsqueda de empleo fuera de la finca, lo que conlleva a una disminución de la mano de obra agrícola local, y al inicio de micro emprendimientos familiares de textiles y artesanías. La juventud rural, consciente de estas limitantes agrícolas, no se siente atraída para continuar con la actividad agrícola, ni tampoco considera estudiar esta ciencia en la formación profesional. La combinación de las problemáticas estudiadas como la migración, el cambio de actividad económica, y de la escasa renovación generacional demuestran la existencia de un proceso de desagrarización de la agricultura familiar indígena, que conllevan a cambios demográficos, económicos y laborales de este grupo de estudio.
\end{abstract}

Palabras clave: migración laboral, desarrollo rural, desagrarización, agricultura de subsistencia, juventud rural

\begin{abstract}
This project studies migration and the generational renewal in the indigenous family farming as part of the process of de-agrarization, from the approaches of indigenous family farming and rural youth in the county of Otavalo. Through quantitative and qualitative analysis of the data gathered with structured surveys, the study determines that this type of agriculture suffers from severe productivity problems that negatively affect the generation of economic income, which encourage rural emigration. Particularly, the decrease in the productivity of family farming is due to environmental factors such as water scarcity and soil degradation. The abandonment of agricultural activities is mainly related to the lack of economic and technical governmental support for family farming, forcing farmers to seek employment outside the farm, which leads to a decrease in the local agricultural workforce, and to the sprout of textile and craft family micro-enterprises. Rural youth, aware of these agricultural constraints, are not attracted to continue with agricultural activity, nor do they consider studying this science in vocational training. The combination of the studied problems such as migration, the change of economic activity, and the scarce generational renewal demonstrate the existence of a process of desagrarización of the indigenous family agriculture, which lead to demographic, economic and labor changes of this study group.
\end{abstract}

Keywords: labor migration, rural development, de-agrarization, subsistence agriculture, rural youth 


\section{Introducción}

La migración afecta al desarrollo de las regiones rurales, principalmente por la pérdida de la fuerza laboral agrícola temporal o permanente y puede afectar a la seguridad alimentaria local y regional, particularmente en países con economías agrícolas (FAO, 2014). Estudios conducidos por FAO \&Van der Geest (2016), determinan que en las regiones de Latinoamérica, Asia Central y Oriente Medio la proporción de la población que depende de la agricultura está decreciendo, particularmente, debido a que al tener economías más diversificadas, ofrecen una gama más amplia de oportunidades de empleo para la población joven. La diferencia entre las tasas de crecimiento de la población agrícola rural y la población rural, usualmente significa que la tasa de empleo y la importancia del ingreso económico fuera de la finca se incrementan, generando un rápido proceso de desagrarización del sector rural en estas regiones. En el marco del sistema de migración contemporáneo, Delgado-Wise y Weltmeyer (2016) argumentan desde una perspectiva política-económica, que la mayoría de migrantes económicos y laborales, migran en respuesta a las condiciones opresivas en sus países de origen a causa del desarrollo desigual generado por el sistema capitalista. Esta migración forzada por disposición, exclusión y desempleo, se genera particularmente por la segmentación y precarización de los mercados laborales a escala global, afectando principalmente a los grupos más vulnerables de la sociedad (Delgado-Wise, 2013).

Particularmente, la globalización ha disminuido las condiciones de vida de la clase trabajadora, clase media, campesinos (Otero, 2006), incrementado la brecha entre pobres y ricos (Delgado-Wise y Weltmeyer (2016), y generado consecuencias ecológicas negativas que terminan afectando mayormente a agricultores y poblaciones rurales, quienes buscan diversificar sus ingresos mediante la migración (Bilsborrow, 2002; Gray, 2009; Piguet, 2010), el empleo fuera de finca y el cambio de actividad económica (Iqbal et al., 2018).

Por otro lado, la especialización y el avance de la agricultura industrial, y las intensificaciones de los procesos de producción agrícola para mejorar la producción del campo, hacen que la agricultura familiar se convierta en una actividad económica de medio tiempo e incluso como en "un pasatiempo" o hobby (Tan et al., 2013). Conjuntamente con la globalización de la cadena de alimentos y el incremento de los precios de los insumos agropecuarios, (Rossi \&
Garner, 2014), la calidad de las condiciones laborales y diferencias salariales (Delgado-Wise \& Martin, 2015) amenazan la subsistencia de la agricultura familiar y los modos de vida rural particularmente en los países latinoamericanos con altos niveles de pobreza rural (Maletta, 2011). Desde una perspectiva general, la agricultura familiar está perdiendo fuerza laboral mientras que la industria acrecienta sus reservas laborales (Bernstein, 2013). La fragmentación eventual de la tierra y el envejecimiento de los agricultores son fenómenos que también afectan a la agricultura familiar de países desarrollados en Europa (Mandryk et al., 2012), Estados Unidos (USDA, 2014), de manera más acelerada ocurre en los países en vías de desarrollo como China (Tan et al., 2013), la región Subsahariana (Deininger y Byerlee, 2012) y en la región Latinoamericana (Carmagnani, 2008).

Considerando la importancia de la agricultura familiar para la erradicación del hambre y la disminución de la pobreza a nivel global, en la contribución a la seguridad alimentaria, al manejo de los recursos naturales y al desarrollo sostenible (van der Ploeg, 2013; IFAD, 2014), se declaró al año 2014 como el año internacional de la agricultura familiar. Sin embargo, los modos de producción agrícola industriales constantemente amenazan a este tipo de agricultura, por ende, la FAO trabaja para fortalecer y reposicionar a la agricultura familiar en el centro de las políticas sociales, económicas y ambientales de las agendas para el desarrollo regional (FAO, 2014). Las unidades de producción agrícola (UPA) familiares más pequeñas, tienen una mayor agrodiversidad y contribuyen mayormente a la sustentabilidad ambiental y a la preservación y conservación de recursos naturales, así como a la conservación de valores y conocimientos tradicionales (Carmagnani, 2008; FAO, 2014; Swaminathan, 2014, van der Ploeg, 2013). La emigración rural en el Ecuador que es generalmente de carácter económico y laboral, se puede incrementar por los actuales cambios ambientales, los cuales podrían incrementar la migración desde las zonas rurales (Gray \& Bilsborrow, 2013).

En el cantón Otavalo, existe una economía étnica transnacional basada en el capital social que persiste a pesar de la globalización, estos migrantes utilizando tecnologías pre-industriales e industriales han forjado un mercado global para la venta de artesanías de bajo costo manufacturadas con la mano de obra familiar (Kyle, 1999). La migración indígena kichwa otavaleña, compuesta característicamente por varones jóvenes, que viajaban vendiendo artesanías y tocando instrumentos musicales andinos en las calles 
de Europa por un periodo de cinco a seis meses, data a partir de la década de los setenta; esto les ha permitido generar flexibilidad y practicidad en la producción de artesanías para turistas locales y para la exportación (Ruiz, 2006). Célleri y Jüssen, (2012) constatan que este grupo étnico dispone de un tipo de capital social que depende de la solidaridad étnica entre sus redes de migrantes, hecho que les ha permitido le permanencia de la migración rural internacional (Maldonado, 2004).

Este trabajo de investigación analiza la migración y el abandono de la actividad agrícola, como elementos de la renovación generacional en agricultura familiar indígena kichwa otavaleña, mediante métodos cualitativos y cuantitativos, desde la perspectiva de la desagrarización en la región andina. Adicionalmente, se propone hallar las principales determinantes que influyen sobre la toma de decisión para la emigración rural, y para el empleo fuera de la finca por parte de los agricultores indígenas, y para la participación en la agricultura familiar por la juventud rural que estudian en el colegio técnico agropecuario perteneciente al cantón. Estos resultados permitirán demostrar y responder desde un estudio particular la hipótesis central de la existencia de un proceso de desagrarización en la agricultura familiar indígena kichwa, además contribuyen con evidencia empírica de un fenómeno social, laboral y agrícola para la formulación de política agrícola y de desarrollo rural en conjunto.

\section{Materiales y métodos}

La información empírica se recolectó mediante encuestas estructuradas cerradas, en la parroquia Miguel Egas Cabeza, también conocida como Peguche, ubicada en el cantón Otavalo, provincia de Imbabura. La selección del lugar se realizó en base a consideraciones sobre el tamaño de la tierra, alta presencia de poblaciones indígenas e instituciones educativas agrícolas o agropecuarias. El cantón Otavalo se localiza a $20 \mathrm{~km}$ de la capital de la provincia de Imbabura, la ciudad de Ibarra. Tiene una extensión de $579 \mathrm{~km}^{2}$, se localiza a una altura de 2.565 m.s.n.m. entre las coordenadas: $0^{\circ} 13^{\prime} 43^{\prime}$ 'N y $78^{\circ} 15^{\prime} 49^{\prime}$ 'O. La población indígena de lengua kichwa acentuada mayoritariamente en el sector rural, compone el 57,24\% de la población del cantón (SIISE, 2013). Según el último Censo Nacional se determina que es un cantón rural por tener el $62,50 \%$ de la población residiendo en las más de 75 comunidades ubicadas predominantemente alrededor del lago San Pablo (INEC, 2010). Se caracteriza por una alta densidad poblacional lo que ha generado continuas experiencias migratorias de carácter nacional e internacional, estableciendo una economía transnacional basada en el capital social, y permitiéndoles introducir con éxito sus productos artesanales y textiles en el mercado turístico (Célleri y Jüssen, 2012). Los estudios sobre los aspectos culturales del desarrollo económico en relación a las dinámicas de la migración kichwa otavaleña, asume una fuerte conciencia étnica y se los conoce como una cultura ejemplar al mantener sus tradiciones y por insertarse con sus artesanías en mercados globalizados, conocidos como nichos económicos (Meisch, 2002). El transnacionalismo empresarial del pueblo kichwa otavaleño (Kyle 2003), conlleva lazos entre los migrantes y el lugar de origen en el que se desarrollan redes familiares, de parentesco y de afinidad étnica evidencia una migración de constante retorno (Célleri y Jüssen, 2012).

\subsection{Muestreo y Encuestas}

Con el fin de relacionar las percepciones a emigrar que tienen los agricultores, con la continuidad de la agricultura por las poblaciones jóvenes rurales, este estudio emplea dos tipos de encuestas dirigidas a los dos grupos de estudio, los agricultores indígenas kichwa y a estudiantes del Colegio Técnico Agropecuario Chávez Reyes ubicado en el cantón. i) la encuesta a nivel de agricultora familiar aplicada a hogares agrícolas de etnicidad indígena kichwa en la parroquia Miguel Egas Cabezas, está compuesta por secciones que reúnen información socio-económica, agrícola y migratoria, ii) la encuesta a nivel de juventud rural fue diseñada para entender la renovación generacional desde la perspectiva de los jóvenes, que reciben una educación y formación agropecuaria en colegios técnicos rurales. Esta encuesta permitió recolectar información socio-demográfica, de participación, secuencia o continuidad con la agricultura familiar y la agricultura como una posible área laboral para la futura formación profesional. En total se recolectaron 196 encuestas a nivel de agricultores y 124 a nivel de juventud rural.

Al existir una diferencia en el tamaño poblacional entre dos grupos de estudio (i.e. comunidad y colegio agrícola) se emplearon metodologías diferentes para la recolección empírica de la información. En el primer caso se utilizó el método bola de nieve para identificar a los hogares agrícolas indígenas kichwa; en el segundo grupo se seleccionó a estudiantes de 
los dos últimos años (2do y 3ro) de bachillerato del colegio técnico agropecuario de la zona.

\subsection{Análisis Estadístico}

Para profundizar con el análisis de los datos descriptivos se pretende emplear métodos estadísticos inferenciales con el fin de llegar a detectar las diferencias significativas entre grupos de hogares que piensan migrar vs. los que no consideran migrar. Mediante la prueba $t$ de distribución student que evalúa estadísticamente las diferencias que existe entre la media de dos muestras se establece que la hipótesis nula de que la media de la población estudiada es igual a un valor especificado $\mu_{0}$, además se trabaja con un nivel de significancia del $5 \%$ y se hace uso del siguiente estadístico:

Donde:

$$
t=\frac{\mathrm{x}-\mu}{\mathrm{s} / \sqrt{\mathrm{n}}} \quad \begin{aligned}
& \overline{\mathrm{x}}=\text { es la media de la muestra } \\
& s=\text { estimados para la desviación } \\
& \text { estándar de la población } \\
& \mu=\text { media de la población } \\
& n=\text { tamaño de la muestra }
\end{aligned}
$$

\section{Resultados}

\subsection{Análisis Descriptivo}

\section{Características socioeconómicas de la población}

Las principales características de la población estudiada demuestran que mayoritariamente el jefe de hogar es masculino, donde un tercio de toda la población se encuentra en condición de iletrado y la tenencia de la tierra es propia para ocho de diez agricultores. En la Tabla 1 se puede apreciar el bajo ingreso económico que se genera de la actividad agrícola familiar en un tamaño promedio de la UPA de 0,31 hectáreas, y determina que un tercio de los hogares no genera ningún ingreso de su actividad económica primaria debido a la baja producción agrícola. En la actualidad el Salario Básico Unificado (SBU) en el Ecuador está establecido en USD\$ 386, el ingreso promedio de la actividad principal es alrededor de USD\$192, lo que representa el 49,7\% del SBU.

Tabla 1. Principales características descriptivas de los agricultores en la agricultura familiar indígena

\begin{tabular}{llc}
\hline & & Otavalo \\
Características & Masculino & $\mathrm{N}=196$ \\
\hline Género (\%) & Femenino & 52,55 \\
\hline & Primaria & 47,45 \\
& Secundaria & 52,04 \\
& Técnica & 12,76 \\
& Universitaria & 0,00 \\
Nivel de educación (\%) & Iletrado & 4,08 \\
\hline & Propia & 31,12 \\
\hline UPA (\%) & Rentada & 82,65 \\
\hline Edad promedio (años) & Prestada & 7,14 \\
Tiempo ejerciendo actividad económica (años) & Comunal & 10,20 \\
Nro. de miembros del hogar (\# personas) & & 0,00 \\
Ingreso económico (USD) & & 45,23 \\
Hogares sin ingreso (\%) & & 34,85 \\
Tamaño UPA (m²) & & 5,72 \\
\hline
\end{tabular}




\section{Características agrícolas de la agricultura fami-} liar indígena

En el reducido tamaño de la UPA donde se desarrolla la agricultura familiar indígena, permite la producción de cultivos tradicionales como el maíz (Zea mays), frejol (Phaseolus vulgaris) y cebada (Hordeum vulgare) (Figura 1). El área restante está compuesta por la vivienda, espacios para la crianza de animales de corral, solares y patios. El limitado tamaño de la UPA limita la producción de alimentos en el hogar, que no son suficientes para la generación de ingresos adecuados. Esto se refleja en los hogares donde la agricultura familiar ya no genera beneficios económicos $(30 \%)$, por lo que para este grupo la agricultura familiar indígena es de subsistencia.

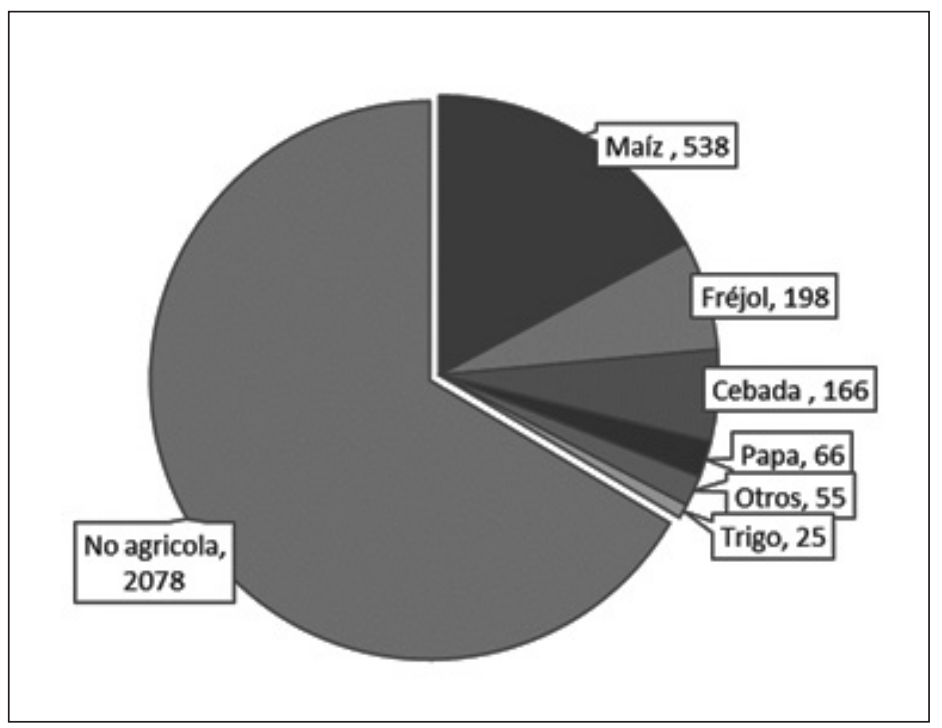

Figura 1. Área de los principales cultivos producidos en la agricultura familiar indígena $\left(\mathrm{m}^{2}\right)$.

\section{Características migratorias}

Este trabajo analiza las variables de migración, abandono de la agricultura y renovación generacional desde los enfoques del agricultor indígena kichwa y de los jóvenes que estudian agricultura, con el fin de entender el proceso de la desagrarización en el cantón Otavalo. El cantón tiene una migración histórica que se origina por la venta de artesanías y exposiciones musicales artísticas a nivel nacional e internacional. El establecimiento de redes de comercio en el Ecuador y de migración en varios países del mundo ha permitido que exista una constante migración del pueblo indígena Otavalo (Meisch, 2002; Ruiz, 2006).

Los resultados demuestran que el abandono de las actividades agrícolas es una opción que está presente en cerca del $17 \%$ de los agricultores. Por otra parte, la migración pasa a ser una alternativa más atractiva para el $25 \%$ de los encuestados, quienes en su mayoría prefieren la migración internacional (Tabla 2).

Tal vez, la mayor preocupación recae en que si, esta agricultura familiar indígena puede subsistir mediante la continuidad y participación de hijos y otros miembros del hogar. En este tenor, se encontró que cerca del $60 \%$ de los agricultores indígenas no cuentan con la participación de alguno de sus hijos en la agricultura, por lo que dependen de otros miembros del hogar para la obtención de la mano de obra que demanda la UPA. Los agricultores kichwa consideran que las duras condiciones en las que se desenvuelve la agricultura, junto a la falta de interés por los jóvenes en este sector y los bajos ingresos que este genera, son las principales causas para el abandono de la actividad agrícola por parte de hijos y jóvenes. Esta ausencia generacional es remplazada en casi el $65 \%$ de los hogares por la mano de obra interna, proveniente del cónyuge del jefe de hogar. Esto ha permitido que el jefe de hogar pueda involucrarse en actividades económicas secundarias fuera de la finca, por medio de la venta de su mano de obra como jornalero agrícola, albañil en el sector de la construcción y la iniciación de emprendimientos textiles y artesanales familiares. 
Tabla 2. Principales características de las percepciones migratorias y continuidad de la agricultura.

\begin{tabular}{lcc} 
Variables & Respuestas & Otavalo \\
\hline \multirow{2}{*}{ Abandonará la actividad económica } & Sí & 16,84 \\
\hline Piensa o va a emigrar & No & $\mathbf{7 0 , 4 1}$ \\
\hline \multirow{2}{*}{ Destino migratorio } & No sabe & 12,76 \\
\hline Hijos trabajando en la agricultura & Sí & 24,49 \\
\hline & No & $\mathbf{7 5 , 5 1}$ \\
\hline Otros miembros del hogar trabajan en la agricultura & Nacional & 22,50 \\
\cline { 2 - 4 } & Internacional & $\mathbf{7 7 , 5 0}$ \\
\hline Miembro del hogar continuará con act. económica & Sí & 42,30 \\
& Nosposo/a & 57,70 \\
\hline
\end{tabular}

Entre las principales consideraciones del agricultor para el abandono de la actividad agrícola, mismas que contribuyen en la formación del proceso de desagrarización, se encuentran la falta de apoyos económico y técnico por parte de instituciones gubernamentales, la presencia de suelos erosionados y los bajos ingresos agrícolas (Figura 2). Estas variables serán analizadas posteriormente mediante métodos estadísticos inferenciales, con el fin de medir la influencia de estas variables predictoras o independientes sobre la influencia en la toma de decisiones para la migración.

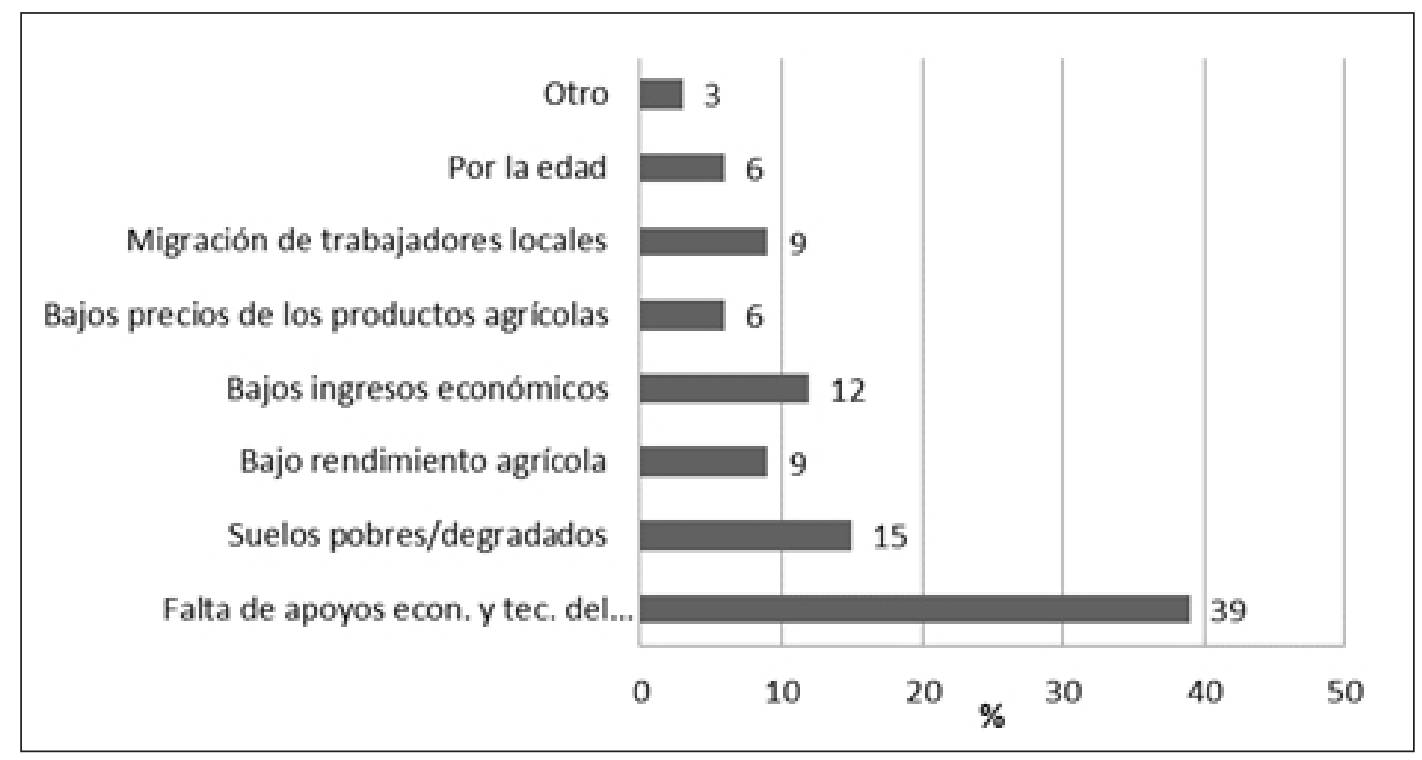

Figura 2. Razones para el abandono de la agricultura. 


\section{Análisis cros-seccional}

Mediante un análisis transversal entre la población de agricultores que piensan migrar y los que piensan lo opuesto, se determina que los agricultores indígenas con edad promedio de 40 años, quienes llevan menos tiempo trabajando en la agricultura, con UPAs más pequeñas y provenientes de hogares más grandes, son propensos a emigrar y abandonar la agricultura familiar. Otra característica, es el tercio de la población que piensa migrar y no genera ningún ingreso económico, debido a que se destina la producción agrícola para el autoconsumo del hogar.

Cabe resaltar que la población que piensa emigrar genera un ingreso agrícola de $20 \%$ adicional, en comparación al grupo que no considera migrar; e incluso es $14 \%$ mayor al promedio total. Esta diferencia de ingresos agrícolas se debe a que los agricultores que van a emigrar trabajan para diversificar las actividades económicas y mejorar los bajos ingresos. Es decir, el 57\% se encuentra realizando una segunda actividad económica temporal fuera de la UPA, con el fin de obtener capital para emprender la migración que mayoritariamente será internacional. Entre las principales actividades secundarias en las que se destacan están la albañilería, jornalero agrícola, emprendimiento de negocios propios, la elaboración de ropa de lana en telares y venta de textiles y artesanías.

Las diferencias significativas de estas variables se determinaron mediante la prueba $t$ para muestras independientes, las distribuciones entre los dos grupos tienen una distribución normal y se comprobó la suposición de la homogeneidad de varianzas vía el test de Levene. Como se puede observar en la Tabla 3 , los agricultores que optarán por migrar $(\mathrm{N}=47)$ están asociados con una edad promedio ( $\mathrm{M}=39,51 \mathrm{años})$ significativamente menor $(\mathrm{p}=0,003)$. Al analizar el tiempo que llevan los agricultores trabajando en la agricultura, se puede establecer que existe una asociación significativa entre los que piensan migrar y los trabajadores que han trabajado un menor número de años en la agricultura. El tamaño del hogar tiene relación significativa con los que aspiran migrar, determinando que provienen de hogares con un mayor número de integrantes. Estos datos revelan el inicio de una transición demográfica que ocurre en esta zona rural al prevalecer los agricultores de mayor edad y al abandonar o emigrar los agricultores jóvenes.

La descripción de los potenciales migrantes de la agricultura familiar sirve para predeterminar escenarios donde la emigración de los agricultores jóvenes y el cambio de actividad económica contribuyen al crecimiento del proceso de desagrarización del campo. Los procesos de migración, abandono y discontinuidad de la agricultura descritos, nos permiten establecer el inicio de un proceso de desagrarización que puede incrementarse eventualmente y llegar a ser irreversible, de persistir estas problemáticas. La inestable situación económica actual en la que se encuentra el Ecuador, las recurrentes amenazas climatológicas y la degradación de los recursos naturales pueden aumentar la vulnerabilidad de esta población rural, forzando principalmente a los agricultores indígenas a la migración y al abandono de la agricultura familiar como actividad económica principal.

Tabla 3. Resultados de la prueba t para muestras independientes entre grupos.

\begin{tabular}{|c|c|c|c|c|}
\hline Variable & $\begin{array}{l}\text { Piensa } \\
\text { migrar }\end{array}$ & Media & $\begin{array}{c}\text { Desviación } \\
\text { Estd. }\end{array}$ & $\begin{array}{c}\text { t-test } \\
\text { Sig }\end{array}$ \\
\hline \multirow{2}{*}{ Edad promedio (años) } & $\mathrm{Si}$ & 39,51 & 17,214 & \multirow{2}{*}{$0,003^{* * *}$} \\
\hline & No & 47,14 & 14,879 & \\
\hline \multirow{2}{*}{$\begin{array}{l}\text { Tiempo ejerciendo actividad económica } \\
\text { (años) }\end{array}$} & Si & 28,35 & 17,399 & \multirow{2}{*}{$0,004 * * *$} \\
\hline & No & 37,01 & 18,240 & \\
\hline \multirow{2}{*}{ Nro. de miembros del hogar (\# personas) } & Si & 6,43 & 2,449 & \multirow{2}{*}{$0,035 * *$} \\
\hline & No & 5,48 & 2,785 & \\
\hline \multirow{2}{*}{ Ingreso económico (USD) } & $\mathbf{S i}$ & 196,73 & 291,679 & \multirow{2}{*}{0,410} \\
\hline & No & 163,61 & 345,400 & \\
\hline \multirow{2}{*}{ Tamaño UPA $\left(\mathrm{m}^{2}\right)$} & Si & 3014,28 & 4968,274 & \multirow{2}{*}{0,884} \\
\hline & No & 3164,81 & 6595,345 & \\
\hline \multirow{2}{*}{ Hogares sin ingreso (\%) } & $\mathbf{S i}$ & 31,92 & - & \multirow{2}{*}{-} \\
\hline & No & 26,53 & - & \\
\hline
\end{tabular}

Nota: $* * *$ altamente significativo $* *$ medianamente significativo, $\alpha=.05$ 
Para determinar la asociación y comparar la distribución actual y esperada de los datos a través de las categorías se realiza un test $C h i$-cuadrado $\left(\chi^{2}\right)$. La tabla 4 demuestra que la relación entre las variables independientes: disminución de la productividad agrícola en los últimos cinco años y consideraciones migratorias es significativa $\chi^{2}(1, N=196)=8.206, p$ $=.004$. Este resultado demuestra que con la disminución de la productividad agrícola (i.e. bajos ingresos) las probabilidades de emigrar se incrementan.

Tabla 4. Resultados de test de Chi-cuadrado para las variables disminución de la productividad y migración

\begin{tabular}{|c|c|c|c|c|c|}
\hline & Valor & df & $\begin{array}{l}\text { Sig. asin. } \\
\text { (2-caras) }\end{array}$ & $\begin{array}{c}\text { Sig. Exacta } \\
\text { (2-caras) }\end{array}$ & $\begin{array}{c}\text { Sig. Exacta } \\
\text { (1-cara) }\end{array}$ \\
\hline Chi-cuadrado de Pearson & $8,206^{\mathrm{a}}$ & 1 & 0,004 & & \\
\hline Corrección de continuidad & 7,269 & 1 & 0,007 & & \\
\hline Razón de verosimilitud & 8,671 & 1 & 0,003 & & \\
\hline Prueba exacta de Fisher & & & & 0,004 & 0,003 \\
\hline Asociación lineal por lineal & 8,164 & 1 & 0,004 & & \\
\hline $\mathbf{N}$ de casos válidos & 196 & & & & \\
\hline
\end{tabular}

a. 0 casillas $(0,0 \%)$ han esperado un recuento menor que 5. El recuento mínimo esperado es de 19,50.

b. Solo se ha calculado para una tabla $2 \times 2$

Para este análisis se emplearon 118 encuestas, descartando 78 encuestas por no estar seguro sobre la posibilidad a emigrar.

La Tabla 5 establece las principales razones que afectan o reducen la productividad agrícola familiar, estas son i) la escasez del recurso hídrico para riego y presencia de sequías temporales (31.4\%), ii) suelos pobres y degradados $(26.3 \%)$ y iii) el ataque de plagas y enfermedades (20.3\%). En la agricultura familiar los costos ambientales por la degradación del suelo se traducen en pérdidas económicas, que vuelven a la agricultura una actividad de baja productividad y poco rentable, en donde se genera una migración rural ambiental y venta de la mano de obra familiar fuera de la finca (Eche, 2013).

Como demuestran los resultados, la disminución de la productividad agrícola es una determinante para la emigración rural, por lo que el incremento de la frecuencia y severidad de estos problemas ambientales y patológicos afectarán negativamente a la producción agrícola y fomentarán más emigración indígena. El crecimiento de este círculo vicioso conformado por la emigración, degradación ambiental y pérdida de productividad agrícola, puede afectar de manera directa sobre la seguridad alimentaria de la población local.

Tabla 5. Tabulación cruzada entre variables disminución temporal de la productividad agrícola y consideración a migrar.

\begin{tabular}{|c|c|c|c|c|c|c|c|c|c|}
\hline & & & \multicolumn{7}{|c|}{ Razón de la disminución de productividad } \\
\hline & & & $\begin{array}{l}\text { Suelos pobres } \\
\text { degradados }\end{array}$ & $\begin{array}{c}\text { Escasez } \\
\text { Agua/sequía }\end{array}$ & $\begin{array}{c}\text { Plagas y } \\
\text { enfermedades }\end{array}$ & $\begin{array}{c}\text { Falta de } \\
\text { asesoramiento }\end{array}$ & $\begin{array}{c}\text { Falta de } \\
\text { apoyo del Gob. }\end{array}$ & Otro & Total \\
\hline \multirow{4}{*}{ 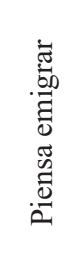 } & $\mathrm{Si}$ & Recuento & 8 & 13 & 10 & 6 & 1 & 0 & 38 \\
\hline & & $\begin{array}{c}\% \text { dentro de Piensa } \\
\text { emigrar }\end{array}$ & 21,10 & 34,20 & 26,30 & 15,80 & 2,60 & 0 & 100 \\
\hline & No & Recuento & 23 & 24 & 14 & 9 & 2 & 8 & 80 \\
\hline & & $\begin{array}{c}\% \text { dentro de Piensa } \\
\text { emigrar }\end{array}$ & 28,80 & 30,00 & 17,50 & 11,20 & 2,50 & 10 & 100 \\
\hline \multirow[t]{2}{*}{ Total } & & Recuento & 31 & 37 & 24 & 15 & 3 & 6 & 118 \\
\hline & & $\begin{array}{c}\% \text { dentro de Piensa } \\
\text { emigrar }\end{array}$ & 26,30 & $31,40 \mathrm{a}<$ & & 12,70 & 2,50 & 5,10 & 100 \\
\hline
\end{tabular}




\section{A nivel de juventud rural}

El abandono del campo y la discontinuidad de la agricultura familiar indígena en Otavalo son dos componentes de un proceso más complejo, que involucra comprender las percepciones de la juventud rural. Los resultados encontrados a nivel de hogar sobre las percepciones a la migración y el abandono de la actividad agrícola de los agricultores indígenas, revelan una parte de la problemática de la desagrarización. Desde otra perspectiva, se consideran a los estudiantes del colegio agrícola local Fernando Chávez Reyes, quienes en la mayoría son hijos de los agricultores de la zona de estudio, esto con el fin de determinar y precisar sobre la renovación generacional y la continuidad de la agricultura familiar indígena. Además, se indaga sobre la posible participación de estos jóvenes en el campo profesional de la agricultura. La figura 3, puntualiza que el trabajo en el sector de la agricultura ya no es atractivo para siete de cada diez jóvenes, este sector sería más atractivo siempre y cuando las condiciones de vida en el campo mejoren, se incremente la productividad agrícola y se generen condiciones laborales más seguras para los agricultores y trabajadores agrícolas.

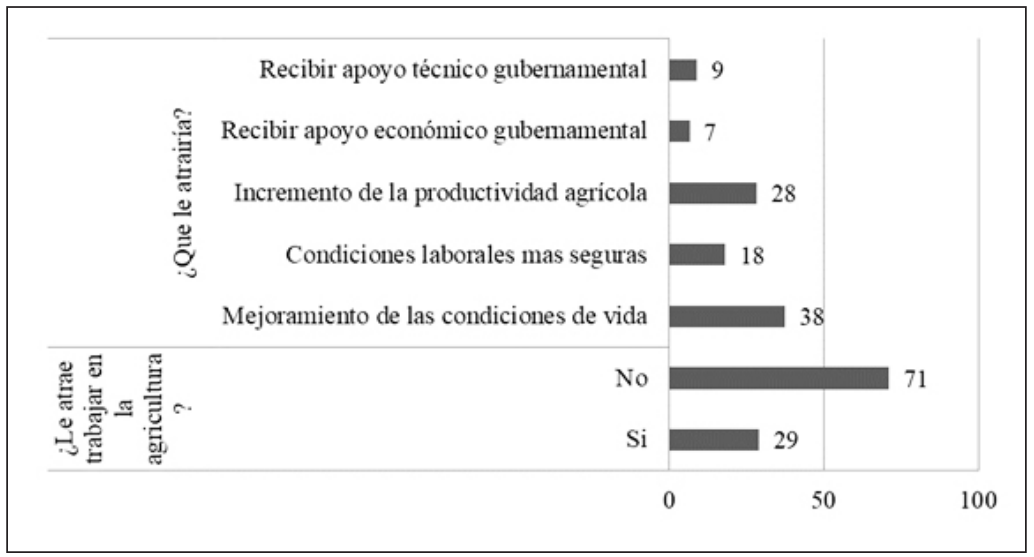

Figura 3. Interés laboral de la juventud rural por la agricultura y determinantes para participar en la agricultura familiar.

Por otra parte, la falta de interés en la agricultura familiar indígena también se refleja en el alto porcentaje de estudiantes del colegio agrícola, que no consideran continuar los estudios de formación profesional en el área de las ciencias agrarias en alguna institución de educación superior del país. La Figura 4 demuestra que las principales razones por la que la agricultura no es una actividad económica rentable para los jóvenes son la baja productividad de la agricultura y los precios bajos de los productos agrícolas. En este contexto, la agricultura no es considerada rentable por el $39 \%$ de los jóvenes encuestados.

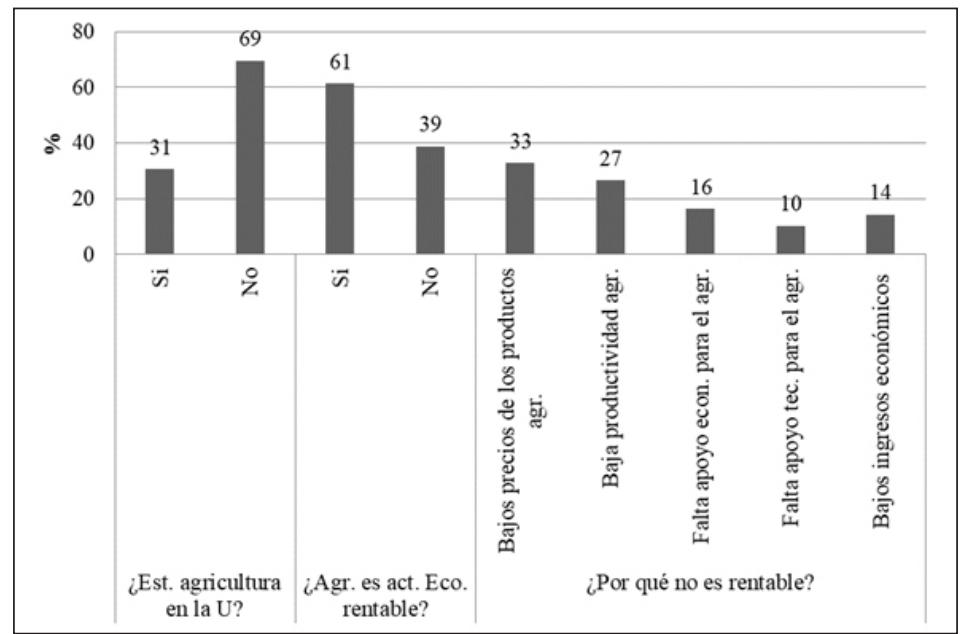

Figura 4. Percepciones de estudio y rentabilidad de la agricultura entre los jóvenes. 
Las conjugaciones de estas y otras condiciones hacen que más de la mitad de los jóvenes consideren abandonar el campo en un futuro cercano, posiblemente después de terminar la educación secundaria. La Fi- gura 5 demuestra que las principales motivaciones para la migración de los jóvenes son los estudios y formación académica en universidades y la búsqueda de mejores oportunidades económicas en las ciudades.

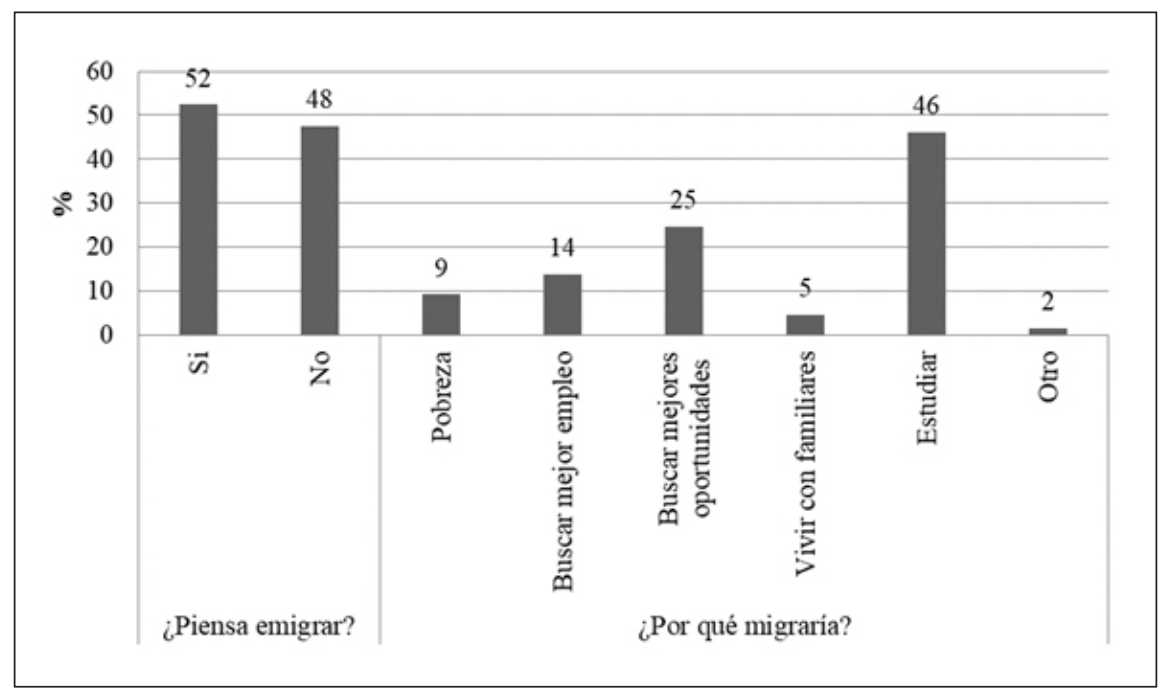

Figura 5. Percepción y razones para la migración por jóvenes

\section{Discusión}

Los resultados del análisis mixto cualitativo-cuantitativo desvelan las características socioeconómicas y agrícolas de la agricultura familiar indígena, que se desenvuelve en un medio rural con limitadas oportunidades de desarrollo. En particular, los bajos ingresos generados por los agricultores se deben principalmente a: i) el tamaño de la UPA que en promedio es de 0,31 hectáreas, lo que refleja la inequidad en la distribución de la tierra en el Ecuador, que en promedio para la agricultura familiar es de 1,4 hectáreas y para las UPAs industriales de 543 hectáreas; además esta agricultura familiar compuesta por más de 535 mil agricultores posee el $6,26 \%$ de la tierra (Brassel, et al.,2008). Las zonas agroecológicas influyen sobre el establecimiento de sistemas agro-productivos, por lo que la marcada topografía definida por los Andes también se ha convertido en una limitante para el tamaño de las UPAs. En contraste, la UPA familiar en Brasil puede tener hasta 440 hectáreas ganaderas y entre 20-50 hectáreas de cultivos (Maletta, 2011). ii) producción limitada de cultivos tradicionales y alimentos básicos (papa, maíz, fréjol); por consiguiente, impactando sobre el ingreso agrícola familiar. Adicionalmente, también se determinó que un 30\% de los agricultores no generan ingresos en la agricultura familiar indígena, al dedicar la producción agrícola al autoconsumo por ser una agricultura de subsistencia. iii) la reducción del agua para riego, y iv) la degradación y erosión de los suelos, tienen un efecto negativo sobre producción, productividad e ingresos agrícolas. Cabe recalcar que, en los países en vías de desarro1lo, la productividad de la agricultura familiar para la generación de alimentos es importante al momento de reducir la pobreza y mantener una adecuada seguridad y soberanía alimentaria (FAO, 2014; Swaminathan, 2014).

Estas determinantes económicas, agrícolas y ambientales se convierten en los principales detonantes de la migración de los agricultores indígenas en el cantón Otavalo. Mientras que la ausencia de apoyos económicos y/o técnicos a la agricultura familiar indígena es el principal determinante para el abandono de la actividad agrícola familiar y la búsqueda de empleo fuera de la finca, estos resultados se corroboran con los resultados obtenidos en otras regiones (Berdegué; Fuentealba, 2011; Carmagnani, 2008). Al considerar las percepciones de abandono de la agricultura (17\%) y a la emigración $(25 \%)$, se observa que, en total cuatro de diez agricultores indígenas dejaran esta actividad económica principal, esto conlleva a un cambio demográfico en la población y eventual desagrarización de la agricultura familiar indígena. Estudios conducidos a nivel de agricultura familiar en el sur del Ecuador corroboran la influencia de la migración en la demografía rural (Bilsborrow, 2002; Gray, 2009; Cé- 
lleri y Jüssen, 2012). Estos cambios demográficos actualmente afectan al $60 \%$ de los agricultores por no contar con la participación de alguno de sus hijos en la agricultura, esta ausencia de mano de obra laboral es sustituida en el $65 \%$ de los hogares por la participación del cónyuge. La falta de renovación generacional ejerce presión sobre la agricultura familiar ya que el $61 \%$ de los hogares no dispone de un miembro del hogar que vaya continuar con las actividades agrícolas en la UPA.

Por otro lado, el $70 \%$ de los jóvenes que estudian agricultura en la institución técnica agropecuaria, no se sienten atraídos a continuar con esta actividad familiar, ni tampoco a estudiar agricultura en la futura formación profesional. Esto a raíz del subdesarrollo en las que se desenvuelve la agricultura familiar indígena, en particular por los bajos ingresos económicos, y duras condiciones laborales. Un estudio en México demuestra que esta problemática se puede resolver con la implementación del trabajo decente en la agricultura (Gálvez et al.,). Con más del 52\% de la población joven con aspiraciones a emigrar, el incremento de la productividad agrícola que conduzca a un mejoramiento de las condiciones de vida (ingreso, salud, educación) es de vital importancia para la prevalencia de la juventud en el campo, la continuidad de la agricultura familiar, el desarrollo rural y contrarrestar el proceso de cambio demográfico agrícola y rural.

\section{Conclusiones}

Este trabajo de investigación al conjugar las perspectivas a la emigración de los agricultores, el abandono de la actividad agrícola, la escasa renovación generacional por algún miembro del hogar y participación de los jóvenes rurales en la agricultura, determinan que existe un proceso de desagrarización de la agricultura familiar indígena. La baja productividad agrícola que es amenazada por la

\section{Recomendaciones}

Brassel, F., Ruiz, P., \& Zapata, A. (2008). La estructura agraria en el Ecuador: una aproximación a su problemática y tendencias. En (eds) Brassel, F., Herrera, S., La Forge, M. ¿Reforma agraria en el Ecuador?: viejos temas, nuevos argumentos. Sistema de Investigación de la Problemática Agraria en el Ecuador (pp. 17-30), SIPAE, Quito- Ecuador. degradación del suelo, escasez crítica de agua-sequías fomenta la migración, mientras que la ausencia de apoyos técnicos y económicos gubernamentales a la agricultura familiar genera el abandono de la actividad agrícola. Estas problemáticas pueden conducir al desaparecimiento y transformación de la agricultura familiar indígena que se la podría catalogar como de subsistencia, por micro-emprendimientos familiares destinados a la elaboración de tejidos en telares tradicionales y ventas de artesanías y textiles en mercados locales e internacionales, al disponer de redes de migrantes establecidas y de capital social.

Adicionalmente, para los agricultores indígenas de este sector, encontrar un sucesor se vuelve cada más difícil y la mano de obra rural es cada vez más escaza, situaciones que producen un cambio generacional y conllevan a una desagrarización del campo. En base a los resultados expuestos se puede elaborar proyectos y planes incluyentes que forjen soluciones a estas problemáticas, mediante la intervención precisa en los elementos que generen el desarrollo rural, particularmente en la agricultura familiar indígena. Es decir, se debe trabajar conjuntamente en i) promover la productividad de la agricultura familiar mediante el diseño, capacitación e implementación de métodos y técnicas bio-intensivas, agroecológicas, orgánicas, para la producción de alimentos que puedan contribuir al mejoramiento del ingreso agrícola, ii) fomentar una transición de la agricultura de subsistencia a una agricultura familiar consolidada, mediante el aprovisionamiento de apoyos económicos y técnicos gubernamentales para la agricultura familiar en particular la indígena, iii) apoyo económico a los agricultores jóvenes para la inversión en la agricultura familiar, iv) mejoramiento técnico e implementación de las TICs para el manejo de la UPA, con el fin de atraer el interés de los jóvenes y modernizar la agricultura familiar.

Berdegué, J.A., \& Fuentealba, R. (2011). Latin America: the state of smallholders in agriculture. In: IFAD Conference on New.

Bernstein, H. (2013). Class dynamics of agrarian change. Stellenbosch: Department of Sociology and Social Anthropology, University of Stellenbosch. 
Bilsborrow, B. (2002). Migration, population change, and the rural environment. Environment Change and Security Program Report, 14(8), 69-94.

Carmagnani, M. (2008). La agricultura familiar en América Latina. Problemas del Desarrollo. Revista Latinoamericana de Economía, 39(153), 11-56.

Célleri, D., \& Jüssen, L. (2012). Solidaridad étnica y capital social. El caso de los comerciantes migrantes Kichwa-otavalo en Madrid y La Compañía. Procesos, 36(2), 143-168.

Deininger, K., \& Byerlee, D. (2012). The rise of large farms in land abundant countries: do they have a future? World Development, .40, 701-714. http:// dx.doi.org/10.1016/j.worlddev.2011.04.030.

Delgado-Wise, R. (2013). The Migration and Labor Question Today: Imperialism, Unequal Development, and Forced Migration. Monthly Review, 64(9), 25. doi:10.14452/mr-064-09-2013-02_3

Delgado-Wise, R., \& Martin, D. (2015). La economía política del arbitraje laboral global. Problemas del Desarrollo, 46(183), 13-32. doi:10.1016/j.rpd.2015.10.002

Delgado-Wise, R. \& Veltmeyer, H. (2016). Introduction. En Veltmeyer, H., \& Delgado-Wise, R., Agrarian Change, Migration and Development (pp. 1-14). doi:10.3362/9781780449166.001

Eche, M. (2013). Land Degradation, Small-Scale Farms' Development, and Migratory Flows in Chiapas. Kassel University Press. Almenia.

FAO. (2014). International Year of Family Farming website. $\square \square$ http://www.fao.org/family-farming-2014/en/ $\square$. Junio 2018.

FAO, \& Van der Geest. (2016). Rural Youth Employment in developing Countries: A Global View. Rural Employment. Roma.

Gálvez, S., Gutiérrez, E., Picazzo, E., \& Osorio, J. (2016). El trabajo decente, una alternativa para reducir la desigualdad en la globalización: el caso de México. Región y Sociedad, 28 (66), 56-94.

Gray, C. (2009). Environment, Land, and Rural Out-migration in the Southern Ecuadorian Andes. World Development, 37(2), 457-468.

Gray, C., \& Bilsborrow, R. (2013). Environmental Influences on Human Migration in Rural
Ecuador. Demography, 50(4), 1217-1241. doi:10.1007/s13524-012-0192-y

IFAD. (2014). International Year of Family Farming: IFAD's Commitment and Call for Action. http://www.ifad.org/events/iyff/ $\square$. Julio 2017.

INEC. (2010). Censo Nacional de Población y Vivienda. INEC-Ecuador

Iqbal, M. A., Abbas, A., Ullah, R., Ahmed, U. I., Sher, A., \& Akhtar, S. (2018). Effect of Non-Farm Income on Poverty and Income Inequality: Farm Households Evidence from Punjab Province Pakistan. Sarhad Journal of Agriculture, 34(2). doi:10.17582/journal.sja/2018/34.2.233.239

Jokisch, D. (2002). Migration and Agricultural Change: The Case of Smallholder Agriculture in Highland Ecuador. Human Ecology, 30(4), 523-550.

Kyle, D. (1999). The Otavalo trade diaspora: social capital and transnational entrepreneurship, Ethnic and Racial Studies, (22)2, 422-446. doi: 10.1080/014198799329549

Kyle. D. (2003). La diáspora comercial de Otavalo: capital social y empresa transnacional. En Portes. A., Guarnizo, L., \& Landolt. P. La globalización desde abajo. Transnacionalismo inmigrante y desarrollo, la experiencia de Estados Unidos (pp. 317), México. Miguel Ángel Porrúa/FLACSO.

Maldonado, G. (2004). Comerciantes y viajeros, Abya-Yala: Ecuador

Mandryk, M., Reidsma, P., \& van Ittersum, M. (2012). Scenarios of long-term farm structural change for application in climate change impact assessment. Landscape Ecology, (27), 509-527. http://dx.doi. org/10.1007/s10980-012-9714-7.

Meisch, L. (2002). Andean Entrepreneurs: Otavalo Merchants and Musicians in the Global Arena. Austin, University of Texas Press.

Otero, G. (2006). México en transición: Globalismo neoliberal, Estado y sociedad civil. México, D.F.: Porrúa

Rossi, J., \& Garner, S. (2014). Industrial farm animal production: a comprehensive moral critique. Journal of Agriculture and Environ- 
ment Ethics, (27), 479-522. http://dx.doi. org/10.1007/s10806-014-9497-8.

Piguet, E. (2010). Climate and Migration: A Synthesis. En Afifi,T., \& Jäger, J. Environment, Forced Migration and Social Vulnerability (pp. 73-85), doi:10.1007/978-3-642-12416-7_6

Ruiz, A. (2006). Espacios de poder transnacional: Diferencias en la migración kichwa-Otavalo. En Santibáñez, R. \& Maiztegui, C. Inmigración: miradas y reflejos. Serie migraciones internacionales, 3(6), 43-58. Universidad de Deusto.

SIISE. (2013). Indicadores sociales del Ecuador, población. Sistema de indicadores del Ecuador. Disponible en http://www.siise.gob.ec/siiseweb/ siiseweb.html?sistema=1\#>. Febrero, 2018.

Snyder, M. (2012). The Family Farm is Being Systematically Wiped Out of Existence in America.
Disponible en http://theeconomiccollapseblog. com/archives/the-family-farm-isbeing-systematically-wiped-out-of-existence-in-america. Marzo, 2018.

Swaminathan, M.S. (2014). Zero hunger. Science 345(6196), 491. http://dx.doi.org/10.1126/science. 1258820 .

Tan, M., Robinson, G., Li, X., Xin, L. (2013). Spatial and temporal variability of farm size in China in context of rapid urbanization. Chinese Geografical Science, (23), 607-619. http://dx.doi. org/10.1007/s11769-013-0610-0

USDA. (2014). Historical Census Data. $\square$ http:// www.agcensus.usda.gov/Publications/Historical_Publications/ $\square$. Agosto 2017

Van der Ploeg, J. (2013). Ten qualities of family farming. Farming Matters, (29), 8-11. 